\title{
A New Stopping Criterion for Efficient Early Termination in Turbo Decoder Designs
}

\author{
Fan-Min Li and An-Yeu (Andy) Wu \\ Graduate Institute of Electronics Engineering, and Department of Electrical Engineering, \\ National Taiwan University, Taipei, 106, Taiwan, R.O.C.
}

\begin{abstract}
Although many stopping methods of iterative decoding have been discussed in the literatures, many of them only focus on the solvable decoding. In this paper, we proposed a new rule, called Measurement of Reliability (MOR) that can be used to define the decoding boundary. With two pre-simulated threshold values, the iterative decoding can stop in either high-SNR situation where the decoded bits are highly reliable, or in low-SNR situation where the decoder already has no capability to decode. Through the simulations, the reduced iterations due to early termination (ET) will not affect the SNR performance. Based on our analysis and simulation results, we can further modify the popularly used GENIE chart. Hence, the iterative decoding can be terminated based on two pre-determined threshold values. The ET property can help to either reduce the computational complexity or power consumptions in implementing the iterative decoding in DSP processors or VLSIs.
\end{abstract}

Keyword: iterative decoding, stopping criterion, early termination, Turbo codes, Turbo principle.

\section{INTRODUCTION}

In 1993, a new class of Forward-Error-Correction (FEC) code, Turbo code, was introduced by Berrou, Glavieux and Thitimajashima [1]. The basic concept of this new decoding scheme is to use a parallel concatenation of at least two codes with an interleaver between the encoders. Decoding is based on alternately decoding the component codes, and passing the so-called extrinsic information (a part of the soft output of the soft-in/soft-out decoder) to the next decoding stage. The soft-output decoding algorithm is usually known as the Maximum A-posteriori Probability (MAP) algorithm [2] [3] or Soft-Output Viterbi Algorithm (SOVA) [4]. The Turbo code is well known for its near Shannon-limit superior decoding performance through iterative processes. Consequently, the $3 \mathrm{G}$ mobile wireless communication system standards [5], such as CDMA2000 and WCDMA, have adopted the Turbo code as the FEC coding scheme. Furthermore, the decoder uses a "turbo" feedback and the method has been generalized to "Turbo-Principle" [6]. It has been successfully applied to many detection/decoding problems such as serial concatenation, equalization, coded modulation, multi-user detection, joint source and channel decoding, etc. Even the rediscovered and extremely good-performance Low Density Parity Check (LDPC) [7] [8] codes are also based on iterative decoding.

As the iterative operations approach the performance limit of a given design, any further iteration results in very little improvement. Therefore, it is important to derive an effective criterion to stop the iterative process, so as to prevent from unnecessary computations and decoding delay. In recent literatures, some stopping criteria for turbo decoders have been presented, and they can be categorized into two classes: One class is based on Soft-bit decisions, such as Cross-Entropy (CE) [9], Mean-Estimation (ME) [10] Updated Threshold [11], and A-priori LLR Measurement [12]. The other class is based on hard-bit decisions, such as Sign-Change-Ratio (SCR) [13], Hard-Decision-Aided (HDA) [13], Sign-DifferenceRatio (SDR) [14], and Improved-HDA (IHDA) [15]. In addition, some methods are based on extra checking policies, such as Cyclic-Check-Codes (CRC) [16] and Valid-Code-Word check (VCW) [17]. However, they only focus on the solvable decoding, that is, the decoder always can recovery correct information bits even with serious noise interference, shown in Fig. 1(a). Obviously, unlimited decoding ability is impossible. Therefore, this paper, we propose a new rule, called Measurement of Reliability (MOR), which considers the decoding boundary. With two pre-simulated threshold values, the iterative decoding can stop in either high-SNR situation where the decoded bits are high reliable, or in low-SNR situation where the decoder already has no capability to decode, as shown in Fig. 1(b). As a result, we can avoid power consumption and decoding delay, and the decoder can be terminated earlier without performance degradation.

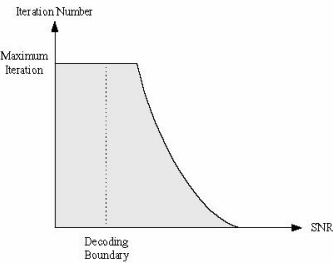

(a)

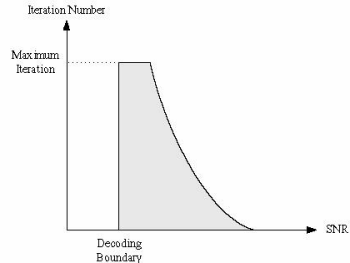

(b)
Fig. 1. Iteration number vs. SNR: (a) Conventional chart (without MOR): The decoder will continue to decoding until the max iteration is reached, even if it is unsolvable under very low SNR. (b) The proposed MOR scheme: The decoder will stop decoding (early termination) when it is unsolvable under very low SNR.

\subsection{High-SNR conditions}

\section{STOPPING CRITERIA}

For different environments, the Turbo decoder can recovery the information bits by iterative decoding. As usual, the decoder must perform more iterative operations in low-SNR channel. For simulations of Turbo decoding, observe the LLR variation vs. iterations, in addition to iterations, it is shown that it

The authors appreciate MediaTek Inc. for their supporting the project. 
grows with iterations and becomes saturated eventually. Because the decoded bits are decided by the LLR, saturated LLR means that it is no longer useful by further iterative decoding. As a result, to avoid power consumption, the decoder can be terminated without performance degradation.

\subsection{Low-SNR conditions}

On the other hand, observe the simulations in Fig. 2 if the decoder can decode successfully in low SNR by given enough iterations. When $E b / N o$ is near $3.8 \mathrm{~dB}$, the decoding result is rapidly changed. Although the decoding is useless as $E b / N o$ is less than $3.804 \mathrm{~dB}$ in Fig. 2(a), it is successful as $E b / N o$ over $3.805 \mathrm{~dB}$ in Fig. 2(b) by operating with more than fifty iterations. In addition, Fig. 3(a) is the simulation of BER vs. iterations relative to Fig. 2(a)(b), and it is shown that there is a decoding boundary of the Turbo decoder, which is near $3.8 \mathrm{~dB}$ Note that there is no fixed and certain generalized boundary for all patterns). Also, observe the simulation in Fig. 3(b), below $E b / N o=3.8 \mathrm{~dB}$, the BER of next iteration is uncertainly better than the one of previous iteration. Therefore, to avoid power consumption, the decoder also can be terminated below the decoding boundary due to useless iterative operations.

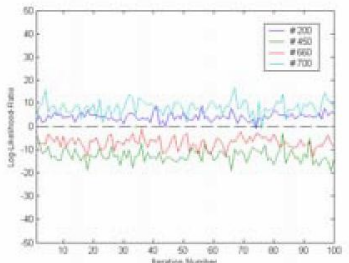

(a)

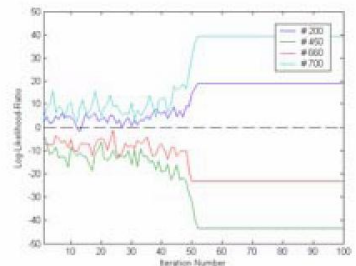

(b)
Fig. 2. Simulations for LLR vs. 100 iterations: (a) $E b / N o=3.804 \mathrm{~dB}$. (b) $E b / N o=3.805 \mathrm{~dB}$.

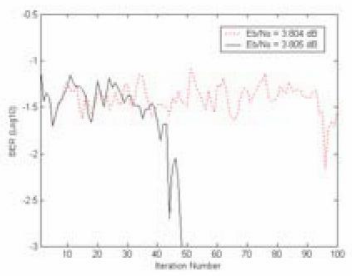

(a)

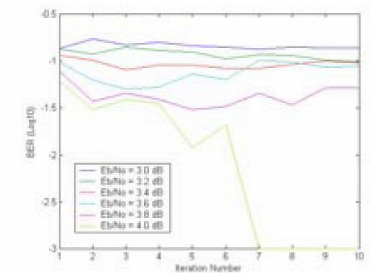

(b)
Fig. 3. Simulations for BER vs. iteration number: (a) $E b / N o=3.804$ $\mathrm{dB}$ and $3.805 \mathrm{~dB}$. (b) $E b / N o=3 \mathrm{~dB}$ to $4 \mathrm{~dB}$

\section{PROPOSED METHODS}

\subsection{Measurement of Reliability (MOR)}

In the Turbo decoding algorithm [1], the magnitude of LLR itself is an index that indicates the reliable degree of decoded information bit. Thus, we define the magnitude of LLR $\left|L_{2}^{(i)}\left(\hat{u}_{k}\right)\right|$ as reliability $R_{k}$ by

$$
R_{k}=\left|L_{2}^{(i)}\left(\hat{u}_{k}\right)\right|
$$

Clearly, the decoding is complete and can be terminated if the reliability of all decoded bits is greater than some threshold value, $T h_{1}$.

$$
R_{k}>T h_{1}, k \in\{1,2, \ldots, N\} .
$$

Besides, if the noise is so larger that the decoding can do nothing, that is, the reliability of all decoded bits is smaller than some threshold value, $T h_{2}$.

$$
R_{k}<T h_{2}, k \in\{1,2, \ldots, N\} .
$$

The decoder can stop decoding and send the automatic-repeat- request (ARQ) message.

\subsection{Frame Testing and Stage Testing}

In both Eqs. (2) and (3), it is shown that the testing condition is based on the overall frame. Thus, the approach needs $2 N$ comparison operations per iteration. By logical relationship, the condition of stopping decoding as the reliabilities of all decoded bits are greater (smaller) than the threshold, can be changed by the condition of continuing decoding as the reliability of any decoded bit is smaller (greater) than the threshold. If the reliability of any decoded bit matches the condition, the one of others need not to be tested again and the comparison can be switched off. As a result, the comparison operations can be reduced.

\subsection{Modification on Existing Methods}

Although there are several approaches discussed above, most of all focus on stopping in high SNR. If the noise is heavy, the decoding usually performs with maximum iterations, even out of the decoding ability. Therefore, some approaches can be modified to stop in low-SNR situation, such as SCR, SDR, and ME methods. Besides, most of all operate based on one overall frame, and some of them also can be modified to operate in stage testing, such as HDA and IHDA methods.

\section{A. Modified-ME (M-ME) [10]}

In addition to the original condition, we add anther condition of stopping in low SNR that

$$
M_{|L|}<T h^{\prime},
$$

where $T h^{\prime}$ is a pre-defined threshold that the mean of absolute LLR can not be greater than in low SNR.

\section{B. Modified-SCR (M-SCR) [13]}

For low SNR, it is shown that the sign of LLR changes rapidly. Therefore, in addition to the original conditio, we add anther condition of stopping in low SNR that

$$
C(i)>T h,
$$

where $T h$ is a pre-defined threshold that the frequency of sign change is greater than in low SNR.

C. Modified-SDR (M-SDR) [14]

Similar to M-SCR, in addition to the original condition, we add anther condition of stopping in low SNR that

$$
D(i)>T h,
$$

where $T h$ is a pre-defined threshold that the frequency of sign change is greater than in low SNR.

D. Modified-HDA (M-HDA) [13]

By logical relationship, the condition of stopping decoding as the signs of all decoded bits are the same as those of previous iteration, can be changed by the condition of continuing decoding as the sign of any 
decoded bit is different from the one of previous iteration. If any decoded bit matches the condition, others need not to be tested again and the comparison can be switched off. As a result, the comparison operations can be reduced.

\section{E. $\quad$ Modified-IHDA (M-IHDA) [15]}

Similar to M-HDA, frame testing can be modified as stage testing. Thus, the comparison operations can be reduced.

\section{SIMULATIONS AND COMPARISONS}

We perform simulations for 4-state Turbo codes, which generator polynomials are $[1,1,1]$ and $[1,0,1]$. The Turbo-codes are obtained for hundreds of frames, where length $N$ is 1000 , maximum iteration is 10 , and an overall rate $R$ is $1 / 2$. The reductions of decoding iterations using different stopping criteria for the iterative Maximum A-posteriori Probability (MAP) [2] [3] decoding scheme are considered.

\subsection{Simulations for GENIE-Aided Benchmark}

Before simulating for above stopping approaches, it needs a GENIE-aided benchmark (The information bits are known and the iteration is stopped immediately after the frame is correctly decoded) to be the performance boundary. Fig. 4 illustrates the equivalent GENIE curve composed of low-SNR and high-SNR situations. The conventional GENIE curve is popularly used in previous stopping approaches. By the simulations shown in Fig. 3(a), it can be found that the decoder exists a decoding limitation near $E b / N_{O}=3.8 \mathrm{~dB}$. Therefore, it is necessary to add auxiliary GENIE curve, which the decoder, below the decoding limitation, just runs an iteration to obtain the LLR for stoppingcriterion testing.

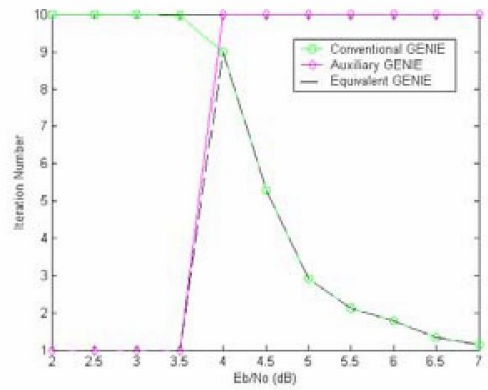

Fig. 4. Simulations for Iteration vs. SNR (GENIE curve).

\subsection{Simulations for Different Stopping Criteria}

With the GENIE curve, the simulations for several approaches discussed above are performed. Fig. 6(a) illustrates the simulations for MOR, SCR, and HDA approaches. It can be found that in high SNR, the performance is close, but in low SNR, only MOR approach has the stopping ability. Then, Fig. 6(b) illustrates the simulations for MOR, SDR, IHDA, and ME approaches. Similar to Fig. 6(a), in high SNR, the performance is close except IHDA, but in low SNR, only MOR approach has the stopping ability. Meanwhile, the IHDA has poor ability for some special frames, which raises the average of iterations. Last, Fig. 6(c) illustrates the simulations for MOR, M-SCR, M-SDR, and M-ME approaches. It can be found that in high SNR, the performance is close, and in low SNR, in addition to MOR approach, others also have the stopping ability.

\subsection{Simulations for Frame Testing vs. Stage Testing}

In the above discussion, we mention that the operations of the MOR approach based on stage testing can be reduced from those based on frame testing. Fig. 5(a) illustrates the simulations for counting the comparison operations of MOR approach. Because the reliability of each decoded bit must compare with two threshold values, the decoding totally includes $2 N$ comparison operations each frame per iteration. Therefore, frame testing will increase the operations with iterations, shown in Fig. 5(a). On the other hand, the stage testing use fewer operations than frame testing on average. By Eq. (2), the decoding terminates, as the reliability of all decoded bits is greater than $T h_{1}$. In other words, the decoding continues, as the reliability of anyone is smaller than $T h_{1}$. In low SNR, the condition is fast matched by such decoded bit. Therefore, observe the stage-testing (condition 1) curve in Fig. 5(a), there are fewer operations in low SNR. Relatively, by Eq. (3), the decoding stops, as the reliability of all decoded bits is smaller than $T h_{2}$. In another word, the decoding continues, as the reliability of anyone is greater than $T h_{2}$. In high SNR, such decoded bit rapidly matches the condition. Therefore, observe the stage-testing (condition 2) curve in Fig. 5(a), there are fewer operations in high SNR. Consequently, the stage-testing (equivalent) curve is the summation of two conditions and keeps smaller than frame testing. Last, we also modified HDA and IHDA approaches in stage testing in Fig. 5(b).

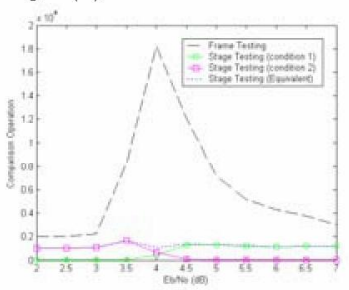

(a)

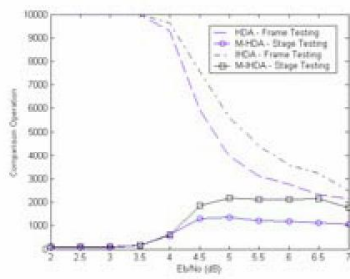

(b)
Fig. 5. Simulations for Comparison Operation vs. SNR: (a) MOR. (b) $\mathrm{HDA}$ and IHDA.

\subsection{Performance Simulations}

Last, the simulations of BER vs. SNR of all approaches described above are performed in Fig. 6(d)(e)(f). Besides, we also add the simulations of conventional GENIE and fixed 10 iterations as the benchmark. It can be found that the performance of them is close either in low-SNR or high-SNR situations, that is, the reduced iterations due to early termination and the saved comparison operations due to stage testing will not affect the SNR performance. 


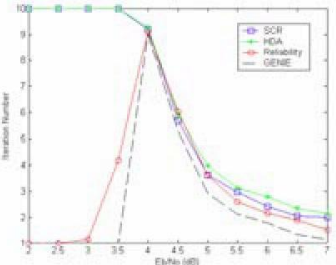

(a)

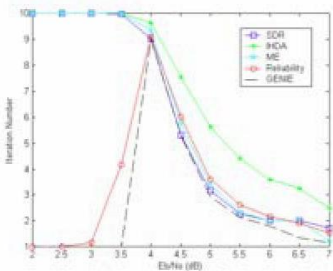

(b)

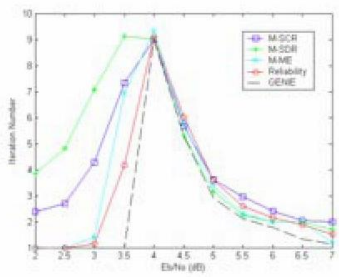

(c)

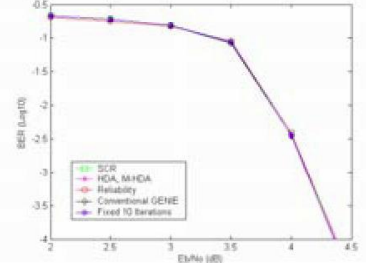

(d)

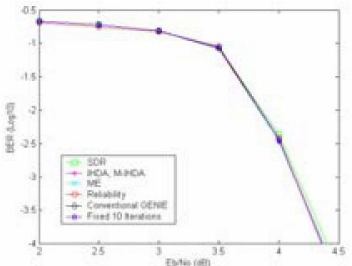

(e)

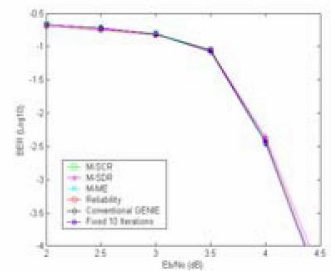

(f)
Fig. 6. Simulations: (a)(b)(c) Iteration number vs. SNR. (d)(e)(f) BER vs. SNR.

\subsection{Summary of ET Approaches}

Last, all stopping methods are summarized in Tab. 1. The main operations include measurement, threshold, and comparing operations. Moreover, due to compare with previous one, some approaches perform after two iterations, such as SCR, HDA, M-HDA, and M-SCR, and they usually need storage. Besides, within the approaches operated with thresholds, their thresholds are usually pre-simulated according to a specific system, even the Updated Threshold method, which one is obtained by pre-simulation and updating with iterations. Last, by the comparison, our proposed MOR approach can perform efficiently with much fewer operations either in high-SNR or low-SNR situations.

\section{CONCLUSIONS}

This paper discusses stopping criteria in high and low SNR channels. We present a new rule, called Measurement of Reliability (MOR). With two presimulated thresholds, the iterative decoding can stop in either high-SNR situation that the decoded bits are high reliable, or in low-SNR situation that the decoder already has no ability to decode. By the simulations, the reduced iterations due to early termination and the saved comparison operations due to stage testing will not affect the SNR performance.

\section{REFERENCES}

[1] C. Berrou, A. Glavieux, and P. Thitimajshima, "Near Shannon limit error-correcting coding and decoding: Turbo codes," in Proc. ICC, pp. 1064-1070, May 1993.

[2] L. Bahl, J, Cocke, F. Jelinck, and J. Raviv, "Optimal decoding of linear codes for minimizing symbol error rate," IEEE Trans. Inform. Theory, vol. IT-20, pp. 284-287, March 1974.

\begin{tabular}{|c|c|c|c|c|c|c|c|c|}
\hline & \multirow{2}{*}{ M. Op. } & \multirow{2}{*}{ T. Op. } & \multirow{2}{*}{ C. Op. } & \multirow{2}{*}{ Storage } & \multirow{2}{*}{$\begin{array}{l}\text { Min. } \\
\text { It. }\end{array}$} & \multicolumn{2}{|c|}{$\begin{array}{l}\text { Stopping } \\
\text { Dem and }\end{array}$} & \multirow{2}{*}{$\begin{array}{l}\text { Test } \\
\text { Unit }\end{array}$} \\
\hline & & & & & & \begin{tabular}{l|} 
High \\
SNR
\end{tabular} & $\begin{array}{l}\text { Low } \\
\text { SNR }\end{array}$ & \\
\hline $\mathrm{CE}[9]$ & $(5 N-1)-\mathrm{R}$ & Pre-sim & $1-\mathrm{R}$ & $(N+2)-\mathrm{R}$ & 1 & $\bigcirc$ & $X$ & Frame \\
\hline SCR [13] & $(3 N-1)-\mathrm{I}$ & Pre-sim & $1-\mathrm{I}$ & $(N+2)$-I & 2 & 0 & X & Frame \\
\hline SDR [14] & $(3 N-1)-\mathrm{I}$ & Pre-sim & $1-\mathrm{I}$ & 1 & 1 & 0 & $X$ & Frame \\
\hline $\mathrm{HDA}[13]$ & $N$-B & - & $N-\mathrm{L}$ & $N$-I & 2 & 0 & $X$ & Frame \\
\hline IHDA [15] & $N$-B & - & $N-\mathrm{L}$ & - & 1 & 0 & $X$ & Frame \\
\hline $\mathrm{ME}[10]$ & $N$-R & Pre-sim & $2-\mathrm{R}$ & 2 & 1 & 0 & X & Frame \\
\hline $\begin{array}{c}\text { Updated } \\
\text { Threshold [11] }\end{array}$ & $N$-R & $\begin{array}{c}\text { Pre-sim, } \\
2-\mathrm{R}\end{array}$ & $2-\mathrm{R}$ & 3 & 2 & 0 & $X$ & Frame \\
\hline $\begin{array}{l}\text { A-priori LLR } \\
\text { Measure [12] }\end{array}$ & $2 N-\mathrm{R}$ & Pre-sim & $1-\mathrm{R}$ & - & 1 & 0 & $x$ & Frame \\
\hline $\mathrm{CRC}[16]$ & $\begin{array}{c}\text { 1-CRC } \\
\text { Decoder. }\end{array}$ & - & $1-\mathrm{L}$ & - & 1 & 0 & $X$ & Frame \\
\hline $\mathrm{VCW}[17]$ & $\begin{array}{l}\text { 1-VCW } \\
\text { Checker }\end{array}$ & - & $1-\mathrm{L}$ & - & 1 & 0 & X & Frame \\
\hline${ }^{*} \mathrm{M}-\mathrm{HDA}$ & $N-\mathrm{B}$ & - & $<N-\mathrm{L}$ & $N$-I & 2 & 0 & $X$ & Stage \\
\hline${ }^{*} \mathrm{M}-\mathrm{IHDA}$ & $N-\mathrm{B}$ & - & $\angle N-\mathrm{L}$ & - & 1 & 0 & X & Stage \\
\hline${ }^{*} \mathrm{M}-\mathrm{SCR}$ & $(3 N-1)-\mathrm{I}$ & Pre-sim & $2-\mathrm{I}$ & $(N+2)$-I & 2 & 0 & 0 & Frame \\
\hline *M-SDR & $(3 N-1)-\mathrm{I}$ & Pre-sim & 2-I & 2 & 1 & 0 & 0 & Frame \\
\hline$* \mathrm{M}-\mathrm{ME}$ & $N-\mathrm{R}$ & Pre-sim & 2-R & 2 & 1 & 0 & 0 & Frame \\
\hline${ }^{*} \mathrm{MOR}$ & - & Pre-sim & $<2 N-\mathrm{R}$ & 2 & 1 & 0 & 0 & Stage \\
\hline
\end{tabular}

[3] P. Robertson, E. Villebrun, and P. Hoeher, "A comparison of optimal and sub-optimal MAP decoding algorithms operating in the log domain," in Proc. Int. Conf. Communications, June 1995, pp. 1009-1013.

[4] J. Hagenauer and P. Hoeher, "A Viterbi algorithm with soft-decision outputs and its applications," IEEE Globecom, pp. 1680-1686, 1989.

[5] R. Steele and L. Hanzo, Eds., Mobile Radio Communications. Second and Third Generation Cellular and WATM Systems, 2nded. New York: Wiley, 1999, ISBN 07273-1406-8.

[6] J. Hagenauer, "The turbo principle: Thtorial Introduction and state of the art," in Proc. Int. Symp. Turbo Codes and Related Topics, Brest, France, 1997, pp. 1-11.

[7] R.G. Gallager, Low-Density Parity Check Codes. Cambridge, MA: MIT Press, 1963.

[8] D. J. C. Mackay and R. M. Neal, "Good codes based on very sparse matrices," in Cryptography and Coding, $5^{\text {th }} I M A$ Conference (Lecture Notes in Computer Science), C. Boyd, Ed. 1995, vol. 1025, pp. 110-111.

[9] J. Hagenauer, E. Offer, and L. Papke, "Iterative decoding of binary block and convolutional codes," IEEE Trans. Inform. Theory, vol. 42, pp. 429-445, Mar. 1996.

[10] F. Zhai and I. Fair, "New error detection techniques and stopping criteria for turbo decoding," in Proc. 2000 Canadian Conference on Electrical and Computer Engineering, vol. 1, pp. 58-62, Mar. 2000.

[11] N. Y. Yu, M. G. Kim, Y. S. Kim, and S. U. Chung, "Efficient stopping criterion for iterative decoding of turbo codes," IEE Electronic Letters, vol. 39, pp. 73-75, January 2003.

[12] D. Bokolamulla and T. Aulin, "A new stopping criterion for iterative decoding," IEEE Commun. Society, pp. 538-541. 2004.

[13] R. Y. Shao, S. Lin, and M. P. C. Fossorier, "Two simple stopping criteria for turbo decoding," IEEE Trans. Commun., vol. 47, pp. 1117-1120, Aug. 1999.

[14] Y. Wu, D. Woerner, and J. Ebel, "A simple stopping criteria for turbo decoding," IEEE Commun. Letters, vol. 4, pp. 258-260, Aug. 2000.

[15] T. M. N. Ngatched and F. Takawira, "Simple stopping criterion for turbo decoding," IEE Electronic Letters, vol. 37, pp. 1350-1351, Oct. 2001.

[16] A. Shibutani, H. Suda, and F. Adachi, "Reducing average number of turbo decoding iterations," IEE Electronic Letters, vol. 35, pp. 701-702, Apr. 1999.

[17] J. Heo, K. Chung, and K. M. Chugg, "Simple stopping criterion for min-sum iterative decoding algorithm," IEE Electronic Letters, vol. 37, pp. 1530-1531, December 2001. 